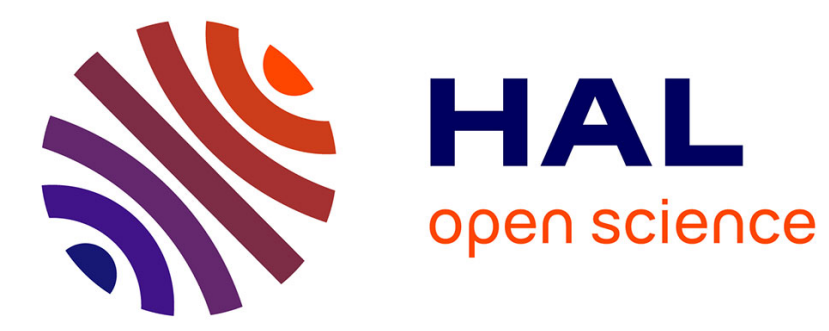

\title{
Clues on the history of early-type galaxies from SDSS spectra and GALEX photometry
}

\author{
A Werle, R Cid Fernandes, N Vale Asari, P R T Coelho, G Bruzual, S \\ Charlot, R R de Carvalho, F R Herpich, C Mendes de Oliveira, L Sodré, et al.
}

\section{- To cite this version:}

A Werle, R Cid Fernandes, N Vale Asari, P R T Coelho, G Bruzual, et al.. Clues on the history of early-type galaxies from SDSS spectra and GALEX photometry. Monthly Notices of the Royal Astronomical Society, 2020, 497 (3), pp.3251-3263. 10.1093/mnras/staa2217 . hal-02989762

\section{HAL Id: hal-02989762 https://hal.science/hal-02989762}

Submitted on 5 Nov 2020

HAL is a multi-disciplinary open access archive for the deposit and dissemination of scientific research documents, whether they are published or not. The documents may come from teaching and research institutions in France or abroad, or from public or private research centers.
L'archive ouverte pluridisciplinaire HAL, est destinée au dépôt et à la diffusion de documents scientifiques de niveau recherche, publiés ou non, émanant des établissements d'enseignement et de recherche français ou étrangers, des laboratoires publics ou privés. 


\title{
Clues on the history of early-type galaxies from SDSS spectra and GALEX photometry
}

\author{
A. Werle, ${ }^{1,2,3 \star}$ R. Cid Fernandes, ${ }^{3}$ N. Vale Asari, ${ }^{3,4} \dagger$ P. R. T. Coelho, ${ }^{2}$ G. Bruzual, ${ }^{5}$ \\ S. Charlot, ${ }^{6}$ R. R. de Carvalho, ${ }^{7}$ F. R. Herpich, ${ }^{2}$ C. Mendes de Oliveira, ${ }^{2}$ L. Sodré Jr., ${ }^{2}$ \\ D. Ruschel-Dutra, ${ }^{3}$ A. de Amorim $^{3}$ and V. M. Sampaio ${ }^{7,8}$ \\ ${ }^{1}$ INAF - Osservatorio Astronomico di Padova, Vicolo dell'Osservatorio 5, 35122 Padova, Italy \\ ${ }^{2}$ Instituto de Astronomia, Geofísica e Ciências Atmosféricas, Universidade de São Paulo, R. do Matão 1226, 05508-090 São Paulo, Brazil \\ ${ }^{3}$ Departamento de Física - CFM - Universidade Federal de Santa Catarina, Florianópolis, SC, Brazil \\ ${ }^{4}$ School of Physics and Astronomy, University of St Andrews, North Haugh, St Andrews KY16 9SS, UK \\ ${ }^{5}$ Instituto de Radioastronomía y Astrofísica, Universidad Nacional Autónoma de México, Morelia, Michoacán, 58089 México \\ ${ }^{6}$ Sorbonne Universités, UPMC-CNRS, UMR7095, Institut d'Astrophysique de Paris, F-75014, Paris, France \\ ${ }^{7}$ NAT-Universidade Cruzeiro do Sul / Universidade Cidade de São Paulo, Rua Galvão Bueno, 868, 01506-000, São Paulo, SP, Brazil \\ ${ }^{8}$ Instituto Nacional de Pesquisas Espaciais/MCT, São José dos Campos, Brazil
}

\section{Accepted XXX. Received YYY; in original form ZZZ}

\begin{abstract}
Stellar population studies of early-type galaxies (ETGs) based on their optical stellar continuum suggest that these are quiescent systems. However, emission lines and ultraviolet photometry reveal a diverse population. We use a new version of the STARLIGHT spectral synthesis code and state-of-the-art stellar population models to simultaneously fit SDSS spectra and GALEX photometry for a sample of 3453 galaxies at $z<0.1$ with $N U V-r>5$ that are classified as elliptical by Galaxy Zoo. We reproduce $F U V$ magnitudes for 80 per cent of UV upturn galaxies selected using criteria from the literature, suggesting that additional stellar population ingredients such as binaries and extreme horizontal branch stars may have a limited contribution to the UV upturn. The addition of ultraviolet data leads to a broadening of the distributions of mean stellar ages, metallicities and attenuation. Stellar populations younger than $1 \mathrm{Gyr}$ are required to reproduce the ultraviolet emission in 17 per cent of our sample. These systems represent 43 per cent of the sample at $5<N U V-r<5.5$ and span the same stellar mass range as other ETGs in our sample. ETGs with young stellar components have larger $H \alpha$ equivalent widths $\left(W_{H \alpha}\right)$ and larger dust attenuation. Emission line ratios and $W_{H \alpha}$ indicate that the ionising source in these systems is a mixture of young and old stellar populations. Their young stellar populations are metal-poor, especially for high-mass galaxies, indicating recent star formation associated with rejuvenation events triggered by external processes, such as minor mergers.
\end{abstract}

Key words: galaxies: evolution - galaxies: stellar content - galaxies: elliptical and lenticular, cD - ultraviolet: galaxies

\section{INTRODUCTION}

Historically, early-type galaxies (ETGs) have been thought of as a more-or-less coeval population of galaxies. Indeed, studies based on their optical stellar continuum are limited in their ability to probe the diversity of this galaxy population. However, studying ETGs in other wavelengths (e.g.

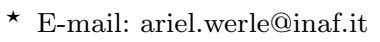

$\dagger$ Royal Society-Newton Advanced Fellowship
Code \& Welch 1979; Kaviraj et al. 2007; Welch et al. 2010; Yi et al. 2011; Simonian \& Martini 2016) or looking closely at emission lines (Herpich et al. 2018) and even specific spectral indices in the optical (e.g. Caldwell et al. 2003) reveals a much more complex and intriguing picture. In particular, studies in the ultraviolet (UV) range have opened an important window to our understanding of these systems.

Some ETGs exhibit a phenomenon called the UV upturn, a strong rise in emission towards the far-UV in galaxies with otherwise red spectra that was first identified by 
Code \& Welch (1979, see review by Yi 2008). Several possible sources have been proposed, associated with several different kinds of stars, the most likely candidates being core helium burning stars in the horizontal branch (HB) and extreme horizontal branch (EHB), and their post-asymptotic giant branch (post-AGB) progeny (e.g. Greggio \& Renzini 1990; Dorman et al. 1993; Yi et al. 1997; O'Connell 1999; Deharveng et al. 2002; Petty et al. 2013; Hernández-Pérez \& Bruzual 2014). Direct evidence of UV emission from resolved hot HB stars was found by Brown et al. (2000b) in the core of the local early-type galaxy M32. Overall, the population of UV upturn galaxies is brighter, redder in the optical, and more massive than other ETGs (e.g. Dantas et al. 2020; Le Cras et al. 2016b, and references therein). There is also evidence for an evolution of the fraction of UV upturn systems with redshift. Brown et al. (2000a) found that the far-UV emission from quiescent galaxies is weaker at $z=0.55$ than at $z \sim 0$. Le Cras et al. (2016b) also find a rise in the frequency of UV upturn systems towards low $z$, in a way that is consistent with the evolution of low-mass stars.

Ultraviolet information also allows the detection of small amounts of young stellar populations in ETGs (e.g. Burstein et al. 1988; Yi et al. 2005; Jeong et al. 2009; Vazdekis et al. 2016). Several works have taken advantage of this feature. Kaviraj et al. (2007), who combined photometry from SDSS and GALEX, find at least some level of young stellar populations (1-3 per cent of stellar mass) in $\sim 30$ per cent of their sample of ETGs. Also using GALEX and SDSS photometry, Schawinski et al. (2007) find that $30 \pm 3$ per cent of massive ellipticals show signs of recent star-formation. Recently, Salvador-Rusiñol et al. (2019) used spectra from the Baryon Oscillation Spectroscopic Survey Dawson et al. (2013) covering the near-ultraviolet range to show that $\sim 0.5$ per cent of the mass of their sample of ETGs was in stars younger than $2 \mathrm{Gyr}$, with the fraction decreasing with stellar mass according to a downsizing pattern. Several other studies also find that the UV colours of some ETGs cannot be explained by old populations alone (e.g. Deharveng et al. 2002; Yi et al. 2011, see also Kauffmann et al. 2007). The detection of young stellar populations is key to address whether the formation of ETGs is consistent with a a monolithic scenario (Larson 1974), in which galaxies form from a single burst of star-formation, or a hierarchical one, where the galaxies undergo significant merging and have a more extended star-formation history (see Kaviraj et al. 2005 and references therein).

Other studies also point to this complex view of earlytype galaxies. Bica \& Alloin (1987) found evidence of young stellar populations in NGC 2865, NGC 4382, and NGC 5102. Recently, Pawlik et al. (2018) found that 40 per cent of massive post-starburst galaxies in the local universe are the result of red sequence galaxies undergoing 'rejuvenation events' and moving towards the massive end of the red sequence through minor mergers. Such rejuvenation of ETGs has also been observed at $z \sim 0.8$ by Chauke et al. (2019). Works by the SAURON collaboration have found that ETGs can be divided in fast and slow rotators (Emsellem et al. 2007) according to the prominence of their rotational component, a classification that has been linked to different formation processes (Cappellari et al. 2011; Penoyre et al. 2017; Smethurst et al. 2018). A recent paper by Carleton et al. (2020) shows a discrepancy between UV and $H_{\alpha}$ star- formation rates in massive galaxies at $z \sim 1$, indicative of low-level bursty star-formation. A series of papers from the SPIDER project also shed light on the diversity of the population of ETGs and the environmental processes involved in their formation (e.g La Barbera et al. 2010, 2014).

The interstellar medium (ISM) of ETGs also provides information on the variety of physical processes taking place in these systems. There is copious evidence of small amounts of cold gas in atomic and molecular form in the ISM of ETGs (e.g. Welch et al. 2010, Agius et al. 2013, Janowiecki et al. 2020, see also Knapp 1999), and the presence of molecular gas have been associated with younger ages (Young et al. 2014). Herpich et al. (2018) have investigated differences between ETGs with and without emission lines. The authors have shown that the galaxies where emission lines are detected also have more $12 \mu \mathrm{m}$ emission (WISE W3 band), attributed to the emission from the policyclic aromatic hydrocarbon (PAH) powered by a hard ionizing field produced by hot low-mass evolved stars (HOLMES; Stasińska et al. 2008; Flores-Fajardo et al. 2011).

In Werle et al. (2019, hereafter W19), we presented a combined analysis of SDSS spectra and GALEX photometry with the STARLIGHT spectral synthesis code (Cid Fernandes et al. 2005, see also López Fernández et al. 2016). This approach allows us to distinguish between different sources of UV emission in ETGs, an asset that was only briefly mentioned by W19. In this paper, we use the W19 synthesis method to revisit previous works on the UV emission of ETGs. In particular, we focus in ETGs in the red sequence $(N U V-r>5)$, which are generally thought of as quiescent systems. Although a lot of work has been done to characterise and study the stellar populations of ETGs in the ultraviolet (Yi et al. 2011; Hernández-Pérez \& Bruzual 2014, to name a few), these works are based on single stellar populations or parametric fits to broad-band measurements of the spectral energy distribution (see Walcher et al. 2011 and Conroy 2013 for reviews), imposing certain assumptions on the star-formation histories of ETGs. Our non-parametric star-formation histories obtained from a combination of full spectral fitting in the optical and photometric constraints in the ultraviolet allow for a more agnostic approach, which is the basis of our contribution to the field.

The paper is organised as follows. Section 2 describes our data and sample. Section 3 details our spectral synthesis procedure and results. In Section 4 we use our synthesis results to explore some open questions in early-type galaxy formation. Finally, conclusions are summarised in Section 5. Throughout this work, we assume a standard $\Lambda \mathrm{CDM}$ cosmology with $\Omega_{\mathrm{M}}=0.3, \Omega_{\Lambda}=0.7$ and $h=0.7$. We adopt the solar metallicity value of $Z_{\odot}=0.017$.

\section{DATA AND SAMPLE}

Here we present a quick review of the data sources used throughout this work and how these data were processed before our analysis.

\subsection{Data sources}

The core analysis of this work relies on a combination of spectra from the seventh data release of the Sloan Digital 
Sky Survey (SDSS DR7; York et al. 2000; Abazajian et al. 2009; www.sdss.org) and photometry from the Galaxy Evolution Explorer (GALEX; Martin et al. 2005).

GALEX provides photometry in two UV bands: $N U V$ $\left(\lambda_{\mathrm{eff}}=2267 \AA\right)$ and $F U V\left(\lambda_{\mathrm{eff}}=1528 \AA\right)$. SDSS spectra typically cover the region between 3800 and $9200 \AA$ in the restframe, with spectral resolution $R \equiv \lambda / \Delta \lambda \sim 1800$. GALEX carried out a series of surveys, the main ones being the AIS (All Sky Imaging Survey), which imaged the whole sky down to a magnitude of 20.5, and the MIS (Medium Imaging Survey), which observed 1000 square degrees of the sky down to magnitude 23. Here we use a combination of data from both surveys.

Although GALEX and SDSS comprise our main data sources, other catalogues are also used throughout this paper. We use emission line measurements from Mateus et al. (2006), galaxy environmental parameters from the catalogue of Yang et al. (2007), morphological information from the Galaxy Zoo project (Lintott et al. 2008) and mid-infrared photometry from the Wide-field Infrared Survey Explorer (WISE, Wright et al. 2010).

\section{$2.2 \quad$ Pre-processing}

Magnitudes used to compute $N U V-r$ and $F U V-N U V$ colours are k-corrected to redshift $z=0$ using the KCORRECT software (Blanton \& Roweis 2007) and corrected for Galactic extinction using a Cardelli, Clayton \& Mathis (1989) extinction law with $R_{V}=3.1$ and $E(B-V)$ values from the dust map of Schlegel et al. (1998), taking into account the re-calibration introduced by Schlafly \& Finkbeiner (2011), i.e. setting $E(B-V)$ to 86 per cent of the value found in the map of Schlegel et al. (1998). GALEX magnitudes used in the STARLIGHT fits were scaled to the SDSS spectroscopic aperture (1.5 arcsec in radius) using the process described in W19.

Spectra are corrected for offsets in the SDSS spectrophotometric calibration by matching synthetic photometry in the $r$-band to observed $r$-band magnitudes in the SDSS spectroscopic aperture. This same procedure was used in the MPA/JHU value-added catalogue ${ }^{1}$. Spectra are then corrected for Galactic extinction using the same method applied to the photometry and, as a last step, are shifted to the rest-frame.

\subsection{Sample selection}

For our core sample, we select galaxies in the SDSS Main Galaxy Sample with $z<0.1$ that were observed in both GALEX bands and are classified as elliptical by Galaxy Zoo. ${ }^{2}$ Since we are interested in red sequence galaxies, we require $N U V-r>5$ for the integrated (i.e. Petrosian) photometry and also for the aperture photometry in a 1.5 arcsec radius, consistent with SDSS spectra. For the aperture photometry, we take into account the aperture correction for

\footnotetext{
1 https://wwwmpa.mpa-garching.mpg.de/SDSS/DR7

2 Although the term "elliptical" is used in the Galaxy Zoo catalogue, this classification includes both elliptical and lenticular galaxies, as stressed by Lintott et al. (2008). Therefore, the correct term to describe galaxies in our sample is "early-type".
}

the GALEX magnitudes described in W19. This constitutes a sample of 3453 early-type galaxies.

\section{MODELLING THE UV-OPTICAL EMISSION FROM EARLY-TYPE GALAXIES}

Reproducing the spectral energy distributions of ETGs while correctly disentangling UV emission from young and old hot stars is challenging, especially when employing nonparametric methods. In this section we describe our method, discuss its limitations and present the main changes in the derived physical properties with respect to optical-only approaches.

\subsection{Spectral synthesis method}

The spectral fits presented in this work are performed with STARLight (Cid Fernandes et al. 2005), a spectral synthesis code that models galaxy spectra through a non-parametric combination of stellar population spectra. The code also fits for stellar kinematics and dust attenuation, assuming a fixed attenuation law. STARLIGHT's fitting procedure is based in a custom implementation of simulated annealing and Markov Chain Monte Carlo that allows the code to capture complex star-formation histories. In this work, we use the version of the code introduced in W19 and in López Fernández et al. (2016), which enables the simultaneous analysis of spectroscopic and photometric data.

The stellar population spectra used in this work are built from an updated version of the Bruzual \& Charlot (2003) simple stellar population (SSP) models (Charlot \& Bruzual, in prep). These models are based on PARSEC isochrones from Chen et al. (2015) (see also Bressan et al. 2012) and a series of stellar libraries, including MILES (Sánchez-Blázquez et al. 2006) and IndoUS (Valdes et al. 2004) in the optical and a blend of theoretical libraries in the UV (e.g. Lanz \& Hubeny 2003a,b; Rauch 2003; Hamann \& Gräfener 2004; Martins et al. 2005; Lanz \& Hubeny 2007; Leitherer et al. 2010, see W19 for more references). In this work, we use models obtained with a Chabrier (2003) initial mass function.

In Fig. 1 we show the evolution in time of several SSP models in the $F U V-N U V$ versus $N U V-r$ colour-colour diagram. Hashed regions in the diagram indicate the criteria used by Yi et al. (2011) to classify ETGs according to their UV emission. We note that in a subsequent section we will present our own criteria to sub-divide the population of ETGs. Solid lines show the evolution of models used in this paper, i.e. those using the post-AGB evolutionary prescription by Vassiliadis \& Wood (1993, 1994, VW hereafter), as described in Bruzual \& Charlot (2003). Models are plotted in the age range from 2 to 14 Gyr (clockwise) for three metallicity values. Dashed lines correspond to the same models but using the post-AGB evolution by Miller Bertolami (2019, and private communication, MB hereafter); these models do not reach the UV upturn regime. Black dots show a solar metallicity model for which the post-AGB evolutionary phase is removed from the tracks and replaced by an $a d-h o c$ contribution of HB stars of increasing temperature, ranging from 8,000 to $30,000 \mathrm{~K}$ (clockwise). The latter models fall in the UV-upturn trapezoid for effective temperatures between 


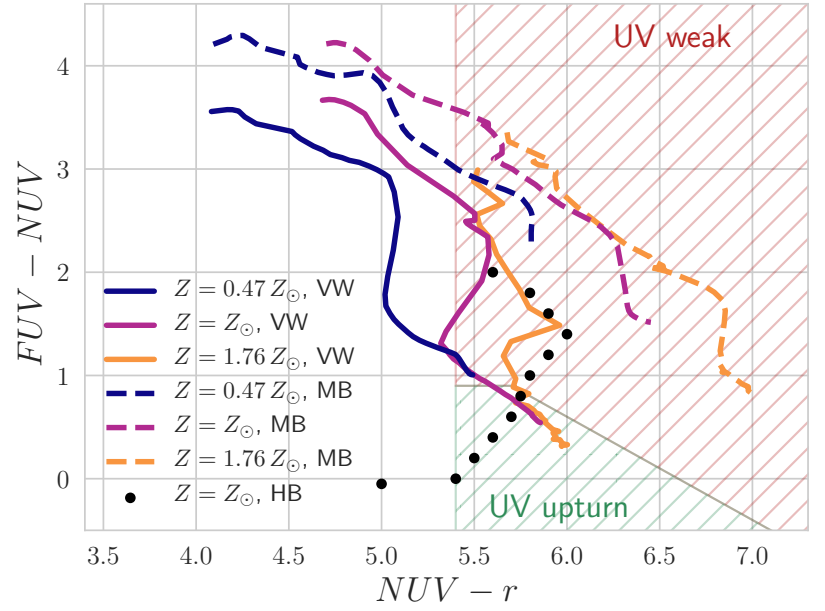

Figure 1. Evolution of simple stellar population models in the $F U V-N U V$ versus $N U V-r$ colour-colour diagram. Models that follow the post-AGB evolutionary prescriptions by Vassiliadis \& Wood (VW, solid lines) and Miller Bertolami (MB, dashed lines) are compared with pure HB models (black dots).

10,000 and 30,000 K. In this paper we adopt the VW models because they follow a physically motivated post-AGB evolution and provide reasonable fits to our data. Using models with HB stars would require updates to the SSP models that are beyond the scope of this paper.

We combine the SSP models in order to build a library of composite stellar populations (CSPs), assuming constant star-formation over a certain period of time. The main difference in this regard between the stellar population models of this paper and the ones from W19 is in how we define the age intervals to build the CSPs. In W19, the CSPs are calculated in time bins that are uniformly sampled in logarithmic space from 0 to $14 \mathrm{Gyr}$. Thus, the oldest stellar population spanned a very wide age range (from $8 \mathrm{Gyr}$ to 14 Gyr). Since here we are interested in spectral features that significantly change in this age range, we split this time interval into five. We also allow for two extra metallicities $\left(Z=1.17\right.$ and $\left.2.35 Z_{\odot}\right)$. These changes are important in order to reproduce the UV slopes of some UV upturn galaxies. The strongest UV upturns are predicted to be in the very old stellar populations (older than $10 \mathrm{Gyr}$ ). Since in W19 we assumed logarithmically spaced age bins, some of these very old stellar populations were averaged with slightly younger (or less old) ones, and had their UV upturns diluted away. Our new base has 180 components, comprising 20 ages and 9 metallicities. We note that throughout this paper we calculate mean stellar ages by assigning to each CSP an age corresponding to the centre of the corresponding age bin.

As in W19, we account for dust attenuation using a Calzetti, Kinney \& Storchi-Bergmann (1994) attenuation law modified in the $\lambda<1800 \AA$ region to smoothly transition to the law of Leitherer et al. (2002). The scheme to combine spectroscopic and photometric figures of merit was kept the same as in W19, as were all other STARLIGHT technical parameters. The choice of parameters is conservative, in the sense that they do not allow STARLIGHT to reduce the quality of the optical fits in order to reproduce the UV.

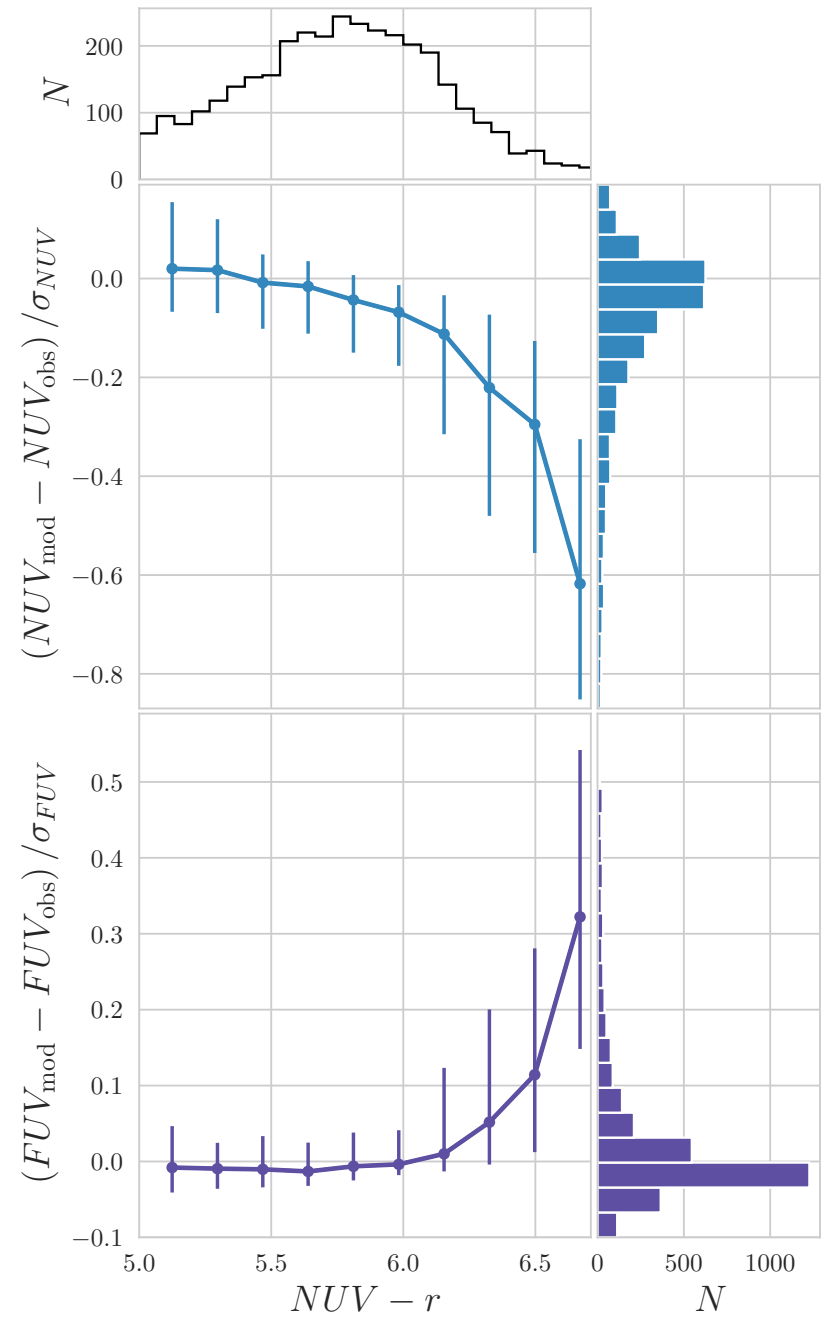

Figure 2. Median residuals in $F U V$ and $N U V$ bands plotted against $N U V-r$ color. Projected histograms show $N U V-r$ color (top), $N U V$ residual (top-right) and $F U V$ residuals (bottomright). Error-bars represent the interquartile regions.

\subsection{Quality of the fits}

To evaluate the quality of our fits across the red sequence, we calculate the UV residuals, defined as the difference between modelled and observed magnitudes divided by the corresponding observational error, and plot them against the $N U V-r$ colour (Fig 2). In general, the fits are remarkably good, although we notice that the quality of the fits decreases for $N U V-r>6$. While the fits remain within error-bars (residuals between -1 and 1 ), one notices a systematic effect where $F U V$ flux is under-estimated and $N U V$ flux is over-estimated. We note, however, that galaxies with $N U V-r>6.5$ are systematically fainter, with $N U V$ magnitudes close to the detection limit of GALEX. As a consequence, sources can be detected only when counts fluctuate above the detection limit. Thus, the bias seen for the $N U V$ band is related to an artifact of the sample selection.

In order to test our ability to reproduce the UV upturn phenomenon, we have selected UV upturn galaxies using the criteria of Yi et al. (2011): $N U V-r>5.4, F U V-N U V<0.9$ 
and $F U V-r<6.6$. These criteria have been originally proposed to separate true UV upturn galaxies from early-type galaxies with residual star-formation, although Dantas et al. (2020) have shown that this cut still allows for a minor contamination from red sequence galaxies with emission lines consistent with residual star formation. In any case these criteria are commonly used in the literature and are useful for comparison. We find that the $F U V$ band is modelled within 0.25 magnitudes in 62.1 per cent of UV upturn galaxies, with 80.8 per cent of the fits within error bars.

This indicates that the old and hot stellar components included in the stellar population models - namely the central stars of planetary nebulae and white dwarfs - can account for the majority of the UV upturn phenomenon in our sample. We interpret that additional components such as binaries (e.g. Han et al. 2007; Eldridge \& Stanway 2012; Hernández-Pérez \& Bruzual 2014) or EHB stars (e.g. Brown et al. 2000a; Petty et al. 2013) are still required to model some galaxies, although their contribution is likely limited overall.

\subsection{Some representative cases}

While ETGs are virtually indistinguishable from each other based on their stellar continuum in the optical, UV information reveals a wide variety of sources within these objects. Such variety, coupled with the incompleteness of the available models and the 'principle of maximum ignorance' (see Cid Fernandes 2007) manifested in STARLIGHT's fitting philosophy, calls for a somewhat artisanal analysis of the dataset. With that spirit in mind, we have visually inspected all of the 3543 spectral fits to evaluate their quality (or lack thereof) and interpret results. Some representative cases of spectral fits are shown in Fig. 3. The left column of panels in the figure shows the UV-optical fit in log scale to highlight the UV, panels in the middle show the optical spectra and panels in the right handside show the FUV population vector, indicating which stellar populations account for the $F U V$ emission in each galaxy (see W19 for details on $\lambda$-dependent population vectors). Each row of panels corresponds to a particular galaxy in our sample.

Fig. 3(a) shows a textbook example of ETG, with flat UV spectrum and only old stellar populations. While in the typical UV-weak ETG (such as Fig. 3a) there is some mixture of different stellar populations, UV upturn galaxies such as the one shown in Fig 3(b) are dominated only by the oldest stellar populations available in the models, which is necessary to simultaneously reproduce their very red $N U V-r$ and blue $F U V-N U V$. For some UV upturn galaxies, the models from our library are incomplete. This is the case shown in panel (c) of Fig 3, where the FUV - NUV colour achieved by STARLIGHT is slightly redder than what is observed. Even allowing only for the oldest stellar populations, the fact that a $\tau_{V}=0.15$ was found for the galaxy in panel (c) can be an indication that STARLIGHT is using dust as a way of forcing the models to become redder in order to reproduce $N U V-r$, which in turn makes $F U V-N U V$ too red. Our inability to fit the $F U V$ band in case (c) could be due to variations in the shape of the attenuation curve, although we deem this unlikely as W19 showed that there is very little variation in the fits obtained with the Calzetti et al. (2000) and Cardelli et al. (1989) laws at low levels of
$\tau_{V}$. Also, we observe a large number of cases where combinations of very old stellar populations with no dust are unable to reproduce $F U V-N U V$ colours, which also leads to the interpretation that these problems are not due to dust, but to the incompleteness in the stellar population models.

Panels (d) and (e) in Fig 3 show two representative cases of ETGs that require some star-formation in the past 1 Gyr to reproduce their integrated light. In case (d), the younger stellar population is not obscured by dust, and the emission lines in the optical indicate only trace amounts of ionised gas. In case (e), the UV colour is relatively red as the young stellar population is very dust-obscured and more mixed with older components than in the previous case. Conspicuous emission lines in the optical indicate that the galaxy retains a fair amount of gas. Based on the emission line diagnostic diagrams (see Fig. 8 in section 4.2), we interpret the source of ionisation in this galaxy to be a mixture of young stars with HOLMES, with a possible contribution from an active galactic nucleus (AGN). A known issue that should be brought up at this point is that the addition of young components may lead to slightly older mean stellar ages measured in the optical. This happens because the young components required to fit the UV make the optical continuum slightly bluer, leading STARLIGHT to invoke slightly older stellar populations to fit the optical continuum, thus reducing the contribution of intermediate ages. One possible solution to this would be to improve our modelling of dust-star geometry. In this work we fit the same dust attenuation for all stellar populations, even though young stars are expected to be more attenuated by dust (e.g. Charlot \& Fall 2000). Introducing extra dust in the young components would have the same effect in the optical as introducing slightly older stellar populations.

\subsection{Changes in physical properties}

After looking at some specific cases, let us now turn to a more statistical approach. As a first step in this direction, we look at the distributions of some physical properties measured by STARLIGHT with and without UV photometry. These distributions are shown in Fig. 4.

Panels (a) and (b) in Fig. 4 show luminosity and mass weighted mean stellar ages, respectively. For fits based purely on SDSS spectra (dashed lines), the distribution of ages is relatively narrow due to two effects: (i) ETGs have very similar optical continuum (see mid panel of Fig. 3), which leads STARLIGHT to assign nearly the same stellar population mixture to all of them, and (ii) as mentioned in W19, STARLight fits to optical spectra tend to misidentify young components at the levels of $\sim 2$ per cent of optical light in all ETGs, which also leads to similar mean stellar ages. This second effect is caused by old hot stars disguised as young components, which is discussed by Ocvirk (2010) and also by Cid Fernandes \& González Delgado (2010). In fits that take GALEX data into account (solid lines in Fig. 4), the information on the UV slope gives STARLIGHT a tool to distinguish between old and young blue stars, removing the fake young components. Also, with UV data we are able to identify differences between stellar populations older than $\sim 7 \mathrm{Gyr}$, which are almost indistinguishable from each other in the optical. This gives us a means to differentiate galaxies that are entirely dominated by the oldest stellar popula- 

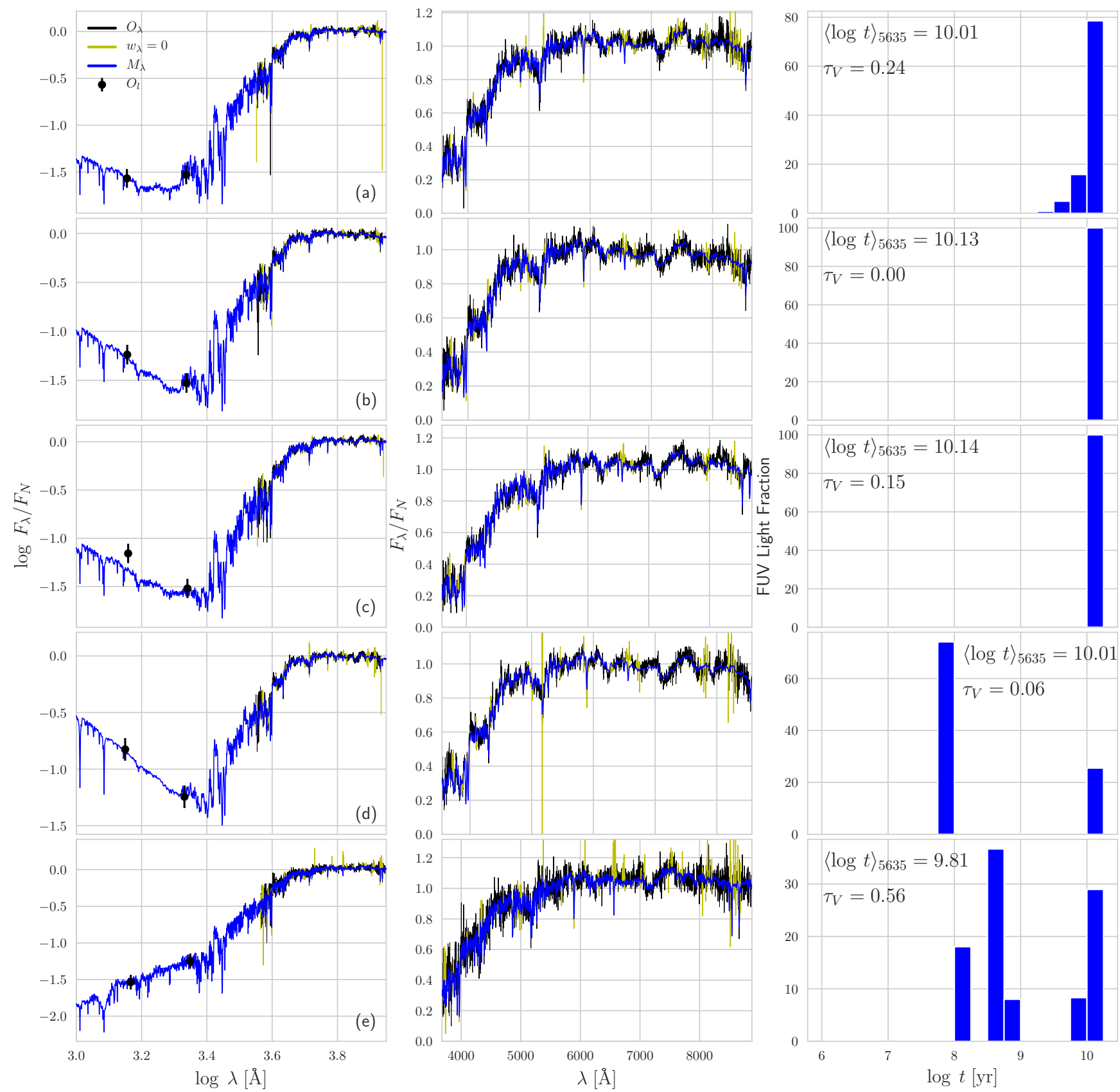

Figure 3. Spectral fits to some representative cases in our sample. Each row of panels corresponds to a different galaxy. Panels to the left show the full UV-optical fit in logarithmic scale and middle panels show a zoom into the optical region. The observed optical spectra $\left(O_{\lambda}\right)$ and UV photometry $\left(O_{l}\right)$ are shown in black. Regions masked out due to the presence of emission lines or sky features are given zero weight $\left(w_{\lambda}=0\right)$ in the fits and are shown in yellow. The modelled spectra $\left(M_{\lambda}\right)$ are drawn in blue. Panels on the right show the $F \boldsymbol{V}$ population vector binned in steps of 0.25 dex in $\log t$, with annotations indicating luminosity-weighted mean stellar ages (at $\lambda=5635 \AA$, $\left.\langle\log t\rangle_{5635}\right)$ and dust optical depth $\left(\tau_{V}\right)$. Fits represent, from top to bottom: (a) a typical UV-weak ETG, (b) an ETG with an UV upturn that is well reproduced by our models, (c) a UV upturn case that is not well reproduced by our models, (d) an ETG that has undergone star-formation in the past 1 Gyr but that does not present strong line emission, and (e) an ETG with recent star-formation, strong dust absorption, and conspicuous line emission in the optical.

tions (Fig. 3b) from others that had more extended periods of star-formation in their early histories (Fig. 3a) and thus slightly younger (or less old) ages. Therefore, this increased sensitivity leads to a broadening of the age distribution.

Metallicities (panels $\mathrm{d}$ and e) remain around the so- lar value when weighted by light and become slightly more metal-rich when mass-weighted. This metal rich population corresponds to galaxies in the upper red sequence to which STARLIGHT assigns old metal-rich components that are redder in $N U V-r$ and bluer in $F U V-N U V$. For some galaxies, 

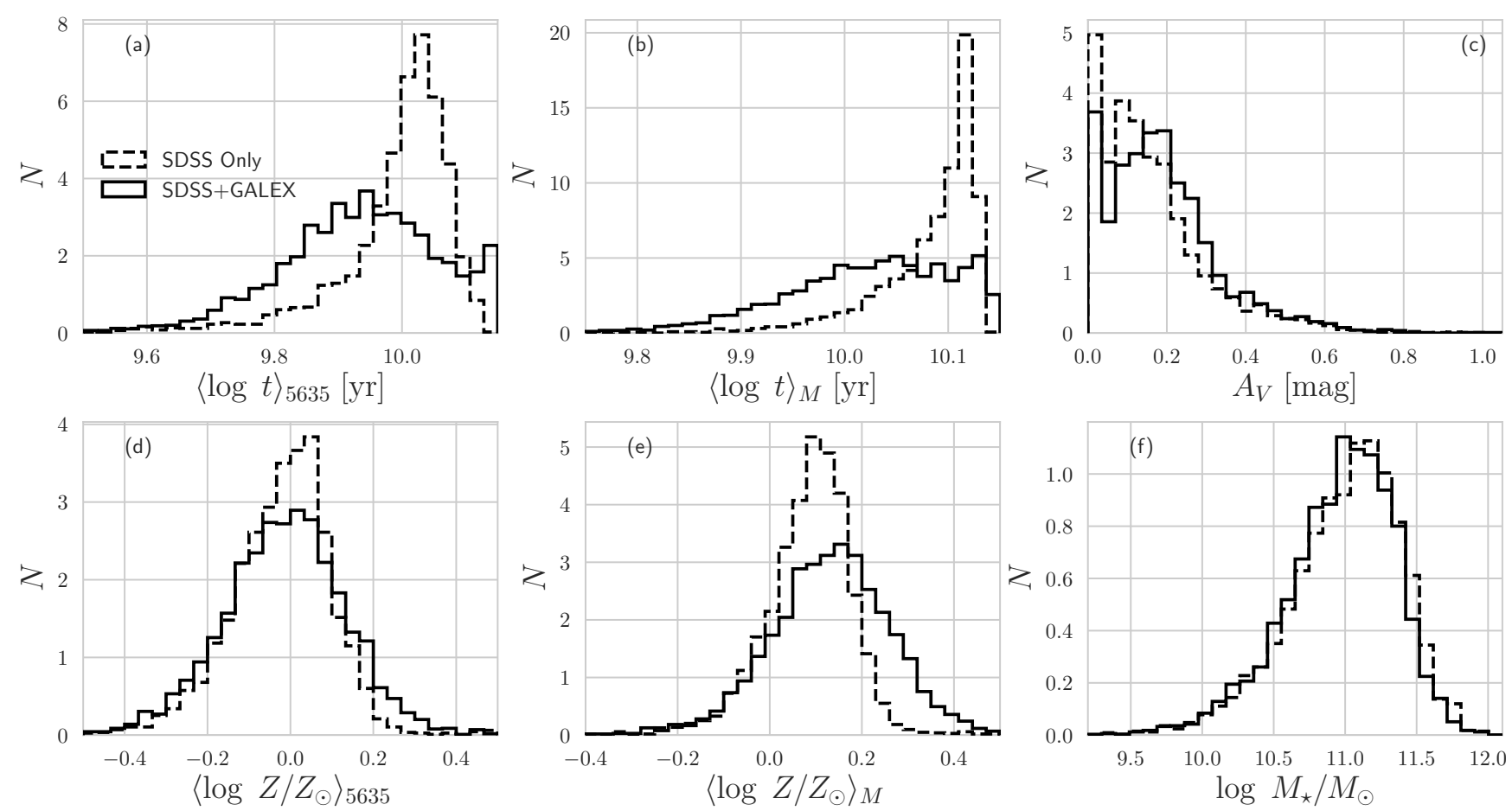

Figure 4. Physical properties measured by STARLIGHT with (solid lines) and without (dashed lines) GALEX data for our sample of ETGs. Histograms are normalized to have the same area. Panels show (a) luminosity-weighted mean stellar ages calculated at $\lambda=5635 \AA$, (b) mass-weighted mean stellar ages, (c) $V$-band dust attenuation, (d) luminosity-weighted mean stellar metallicities calculated at $\lambda=5635 \AA$, (e) mass-weighted mean stellar metallicities and (d) stellar masses. Mean stellar ages are calculated by assigning to each CSP an age corresponding to the centre of a given time bin.

the addition of UV information increases the $V$-band dust attenuation $\left(A_{V}\right.$, Fig. 4c), and for the most part these are ETGs with young stellar components, which will be the subject of sections 4.2 and 4.3. No change is observed in the stellar mass estimates, which is expected since the bulk of the stellar mass is accounted for by populations that emit most of the optical light.

\subsection{Trends with UV colours}

Let us now look more directly at which stellar populations contribute to ultraviolet emission as a function of UV colours. To probe this, we reduce the dimensionality of the $F U V$ population vector, introducing the $F U V$ weighted mean stellar ages and metallicities defined as

$$
\langle\log t\rangle_{F U V}=\sum_{j} x_{j}^{F U V} \log t_{j}
$$

and

$\langle\log Z\rangle_{F U V}=\sum_{j} x_{j}^{F U V} \log Z_{j}$

where $x_{j}^{F U V}$ is the fraction of the galaxy's luminosity in the $F U V$ band that is associated with a stellar population of age $t_{j}$ and metallicity $Z_{j}$. These quantities allow us to easily distinguish between sources of UV emission in ETGs. The definitions above are analogous to STARLIGHT's luminosityweighted mean stellar ages and metallicities used in a series of previous works (e.g. Cid Fernandes et al. 2005; Mateus et al. 2006; Cid Fernandes et al. 2013), but using the FUV population vector to set the weights, thus measuring the properties of the populations that account for $F U V$ emission. In Fig. 5 we plot $\langle\log t\rangle_{F U V}$ (top) and $\langle\log Z\rangle_{F U V}$ (bottom) against $F U V-N U V$ for our sample, colouring points according to $N U V-r$. We note that average uncertainties in luminosity-weighted mean stellar ages and metallicities measured by STARLIGHT are of 0.10 to $0.15 \mathrm{dex}$ (Cid Fernandes et al. 2014). Typical errors in the colours are 0.15 and 0.3 magnitudes for $N U V-r$ and $F U V-N U V$, respectively.

Let us now consider the upper panel of Fig. 5. On the left of the plot are galaxies with young $\langle\log t\rangle_{F U V}$, where UV emission is mostly due to stellar populations younger than 1 Gyr. These systems have relatively steep UV slopes, as measured by $F U V-N U V$, and are more common in the lower red sequence $(N U V-r \lesssim 5.5)$. More quiescent systems form a sequence on the right, with increasingly older $\langle\log t\rangle_{F U V}$ as $F U V-N U V$ becomes bluer. This trend arises because larger contributions of post-AGB stars, and thus older stellar populations are required to model galaxies with blue $F U V-$ $N U V$ and red $N U V-r$. Galaxies in the bottom-right corner of the plot are the ones for which the UV upturn phenomenon is more prominent.

On the lower panel of Fig. 5, one can see that the young stellar populations required to fit the UV emission from galaxies in the lower red sequence are also of low metallic- 

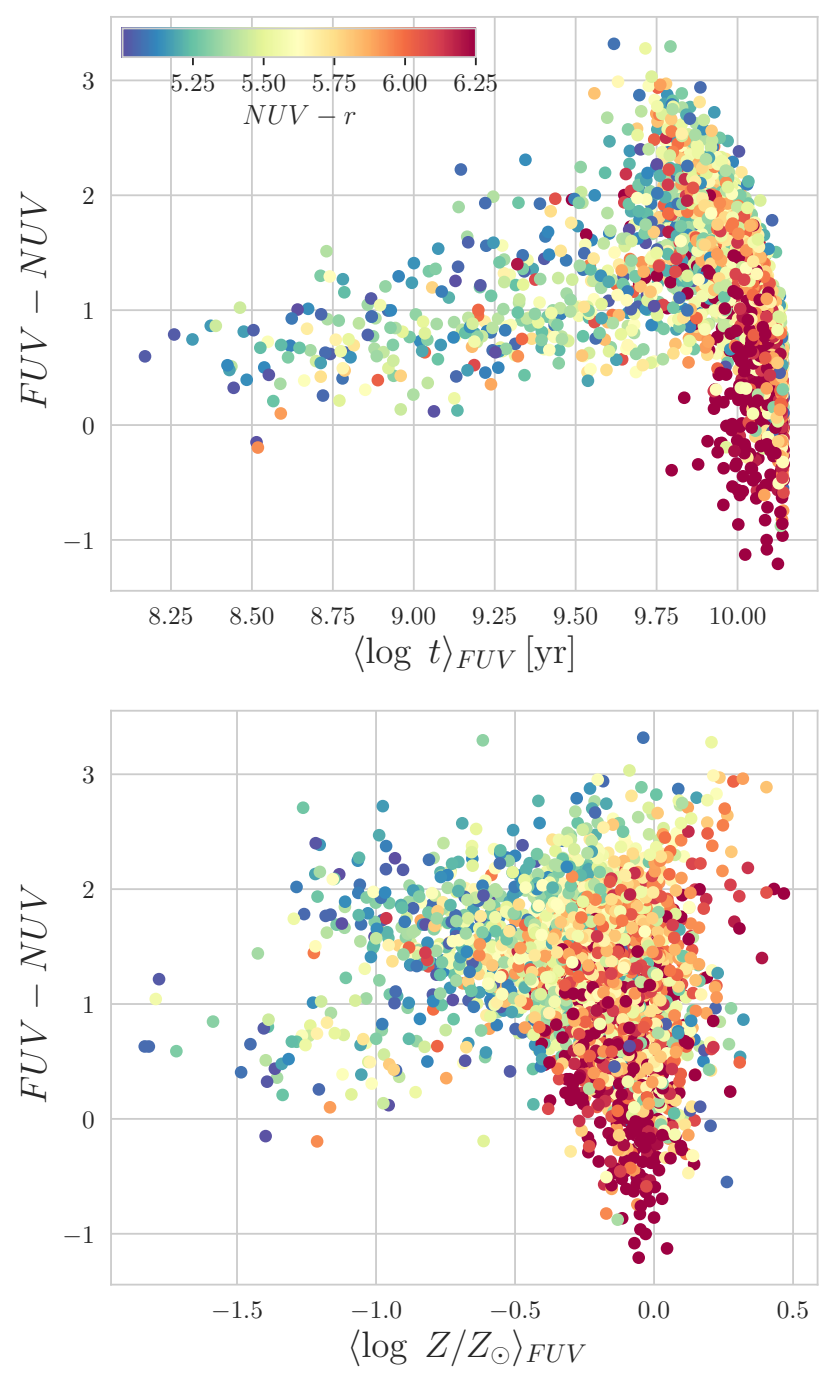

Figure 5. $F U V$-weighted mean stellar ages (top panel) and metallicities (bottom panel) of ETGs in our sample plotted against $F U V-N U V$ with points colour-coded according to $N U V-r$. Colour saturates at $N U V-r=6.25$

ity. This indicates that chemical enrichment is still ongoing on these systems, either through gas recycling or absorption of less chemically evolved galaxies through mergers. In contrast, systems with steep UV slopes (traced by $F U V-N U V$ ) in the upper red sequence are the most metal-rich.

\section{CLUES ON THE HISTORY OF EARLY-TYPE GALAXIES}

We have shown that the addition of UV data reveals the complexity in the population of red sequence galaxies. This opens up the possibility to explore issues that cannot be tackled with optical data alone. In this section, we will use our synthesis results to explore some open questions in earlytype galaxy formation.
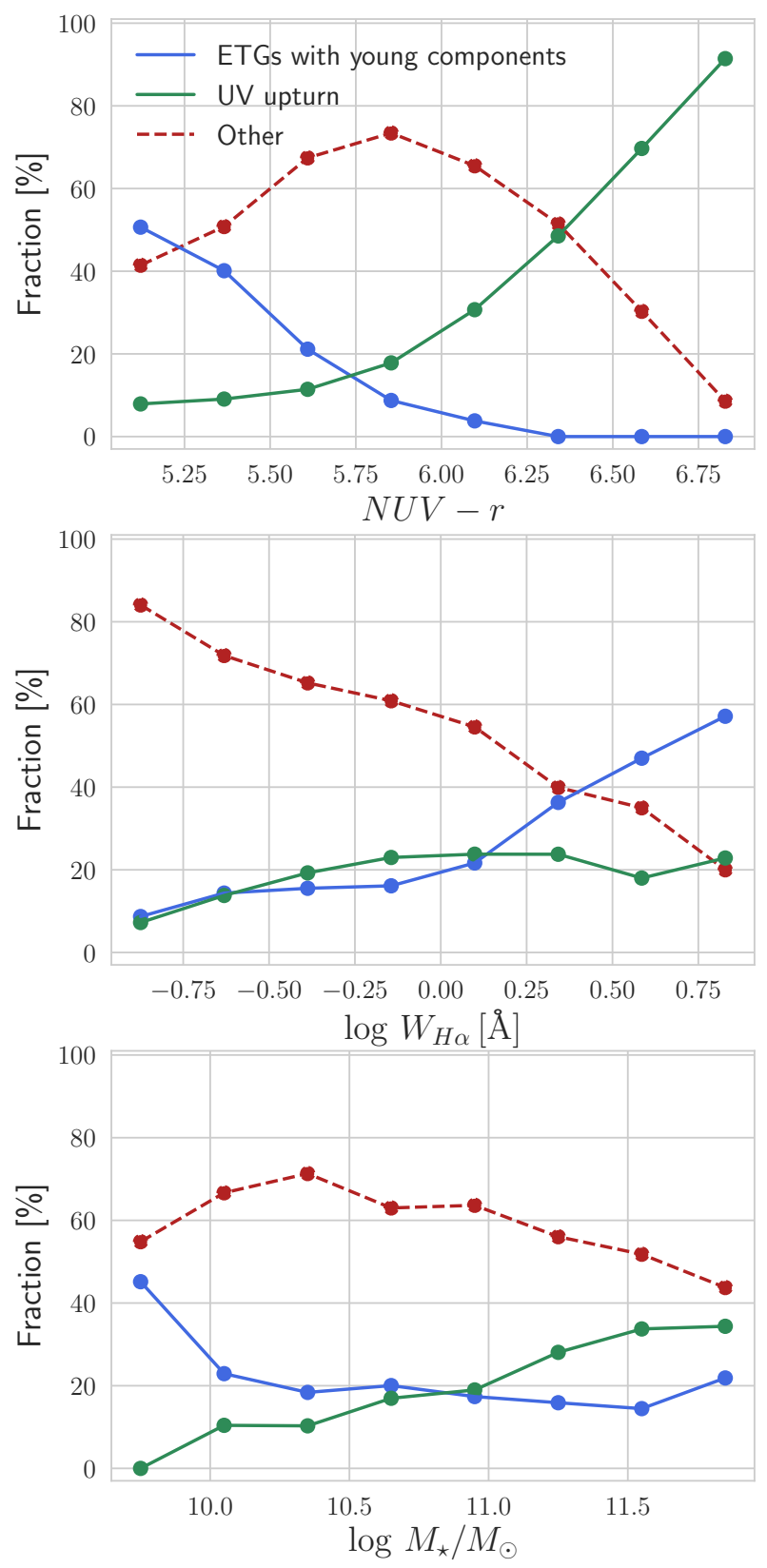

Figure 6. Fraction of galaxies with young components (blue lines), UV upturn systems (green lines), and other ETGs (red dashed lines) in bins of $N U V-r$ (top), equivalent width of $H \alpha$ (middle) and stellar mass (bottom). Points indicate the centre of each bin. In the middle panel, we plot only galaxies with $S / N>3$ in $\mathrm{H} \alpha$.

\subsection{Sub-classes of ETGs according to their UV emission}

As previously mentioned, ETGs can be divided into three groups with respect to the stellar populations that contribute to their UV emission: (i) ETGs where young stellar populations are required to explain the UV (e.g. Fig. 3d and e), (ii) UV upturn galaxies where the very old populations dominate the UV emission (e.g. Fig. 3b and c), and (iii) "intermediate cases" that do not have young components but 
are also not completely old as UV upturn galaxies, i.e. their early star-formation histories are more extended (e.g. Fig. $3 a)$. We thus use the following criteria to identify these subclasses in our sample. (1) We define galaxies as having young components when at least 5 per cent of the $F U V$ emission comes from populations younger than $1 \mathrm{Gyr}$. We find 610 galaxies satisfying this criterion, corresponding to 17.6 per cent of our sample. (2) UV upturn galaxies are defined as the ones with $\langle\log t\rangle_{F U V}>9.75, F U V-N U V<0.9$ (this last criterion is also used by Yi et al. 2011) and no young components, i.e. galaxies in the bottom right of the top panel of Fig. 5. We identify 748 objects (21.6 per cent of our sample) as UV upturn galaxies. (3) Other ETGs, which do not fall in either of the previous categories, make up the remaining 2095 galaxies, 60.6 per cent of our sample.

Our definition of these sub-classes is in reasonable agreement with other results/criteria found in the literature. Kaviraj et al. (2007) found contributions of stellar populations younger than $1 \mathrm{Gyr}$ in 30 per cent of their sample, which includes systems with $N U V-r<5$ where recent starformation is more likely. Considering this caveat, our numbers are in qualitative agreement with those authors. As for the UV upturn sub-class, were we to use the Yi et al. (2011) criteria, we would identify 546 objects ( 16 per cent of the sample) as UV upturn, out of which 396 (53 per cent) also fall into our UV upturn sub-class. We note that we detect young components in 17.5 per cent of the galaxies classified as UV upturn using the Yi et al. (2011) criteria.

The fraction of galaxies in each sub-class varies with respect to galaxy properties. To investigate these variations, we looked at the fractions of each of the sub-classes in bins of $N U V-r$, equivalent width of $\mathrm{H} \alpha\left(W_{\mathrm{H} \alpha}\right)$ and stellar mass $\left(M_{\star}\right)$. Results are shown in Fig. 6. The top panel shows the fraction of each of the sub-classes in bins of $N U V-r$. We find that ETGs with young components make up a significant part of our sample at $N U V-r<5.5$, reaching negligible numbers above $N U V-r=6$. More precisely, the fraction of galaxies with young components is of 43.3 per cent at $5<$ $N U V-r<5.5$ and only 9.5 per cent above $N U V-r=5.5$. UV upturn systems are very rare in the lower red sequence and become the dominant class in the redder $N U V-r$ bins. As in previous studies (e.g. Dantas et al. 2020) we find that UV upturn galaxies are more common in the upper red sequence; in fact, most galaxies with redder $N U V-r$ bins are of this class.

In the middle panel of Fig. 6 , we look at results in terms of $W_{\mathrm{H} \alpha}$. We plot all galaxies with $S / N>3$ in $\mathrm{H} \alpha$ : 73.3 per cent of ETGs with young components, 55.6 per cent of UV upturn galaxies and 53.9 per cent of other ETGs satisfy this criterion. We find that systems with young components represent a significant fraction of our sample at the largest $W_{\mathrm{H} \alpha}$ values, hinting that these systems have more ionized gas than the typical ETG. One should note that, even at the largest values of $W_{\mathrm{H} \alpha}$, a significant fraction of ETGs do not require contributions of young stellar populations to reproduce their UV emission. This should be taken as a word of warning to the use of $\mathrm{H} \alpha$ as an indicator of star-formation in these systems at the SDSS resolution. The sources of ionising photons in these galaxies will be discussed in more detail in the following section. The fraction of UV upturn systems remains almost constant in all bins of $W_{\mathrm{H} \alpha}$, indicating that the UV upturn has no relation with the gas content of the galaxy.

In the bottom panel of Fig. 6 we show results in terms of stellar mass. The fraction of ETGs with young components is larger in the lower $\log M_{\star}$ bin. In this mass range the young stellar populations are also more prominent, and younger ages can be identified from optical spectroscopy (e.g Caldwell et al. 2003), while at large masses UV information becomes crucial to identify recent events of star formation. To be more specific, the $t<1$ Gyr components that we detect correspond, on average, to 0.8 per cent of stellar mass at $\log M_{\star} / M_{\odot}<10.5,0.27$ per cent at $\log M_{\star} / M_{\odot}>10.5$ and $\sim 0.35$ per cent overall. The larger incidence of young components at lower masses is consistent with a downsizing effect, as in the low-mass end of the red sequence galaxies are expected to have quenched their star-formation more recently (e.g Gonçalves et al. 2012; Rowlands et al. 2018). However, it is interesting to note that the downsizing signature is small, and the fraction of these objects remains almost flat for $\log M_{\star} / M_{\odot}>10$. The fraction of UV upturn galaxies rises steadily towards larger values of $\log M_{\star}$, which is in agreement with several works that find the UV upturn to be more common in high-mass galaxies (e.g. Burstein et al. 1988; Smith et al. 2012; Le Cras et al. 2016b; Dantas et al. 2020).

\subsection{The ISM of ETGs with young components}

We detect signatures of stellar populations younger than $1 \mathrm{Gyr}$ in a significant fraction of galaxies at all stellar masses. A similar result was found by Salvador-Rusiñol et al. (2019) at $0.35 \leqslant z \leqslant 0.6$. Despite differences in the methodology, the similarity between their results and ours shows that massive galaxies must be undergoing intermittent star-formation event from $z \sim 0.6$ to $z<0.1$. Several studies also find that cold gas is available in atomic and molecular form in ETGs of all stellar masses (Welch et al. 2010; Young et al. 2014; Castignani et al. 2020). The molecular gas fractions are found to be related to both star-formation histories (Young et al. 2014) and kinematics (Cappellari et al. 2013), in that galaxies with rotating stellar disks have larger gas fractions. Ionised gas can also be detected in the ISM of ETGs of all masses (Herpich et al. 2018). In this section, we will investigate the connection between the presence or absence of young components and the ISM properties of ETGs.

In Fig. 7, we plot $W_{\mathrm{H} \alpha}, A_{V}$ and the WISE colour $W 2-W 3$ against stellar mass for ETGs with (blue lines) and without (red lines) young components. In the top panel of Fig. 7, we plot only galaxies with $S / N>3$ in $\mathrm{H} \alpha$, while in the bottom panel we exclude 3 galaxies for which WISE data are not available, all of them with no young components. We have shown in Fig. 6 that most galaxies in our sample that have $\log W_{\mathrm{H} \alpha}>0.5 \AA$ require $t<1$ Gyr stellar populations to explain their UV emission. In the top panel of Fig. 7, we show that these systems have larger $W_{\mathrm{H} \alpha}$ regardless of stellar mass. ETGs with young components also have larger dust attenuation. This can be seen in terms of the $V$-band dust attenuation derived with STARLIGHT (middle panel) and also in the $W 2-W 3$ colour (bottom panel). The WISE $W 3$ band includes the 11.2 and $12.7 \mu \mathrm{m}$ PAH features. Thus, excess emission in this band (redder $W 2-W 3$ ) indicates the presence of dust. The results in Fig. 7 are comparable to the ones of 


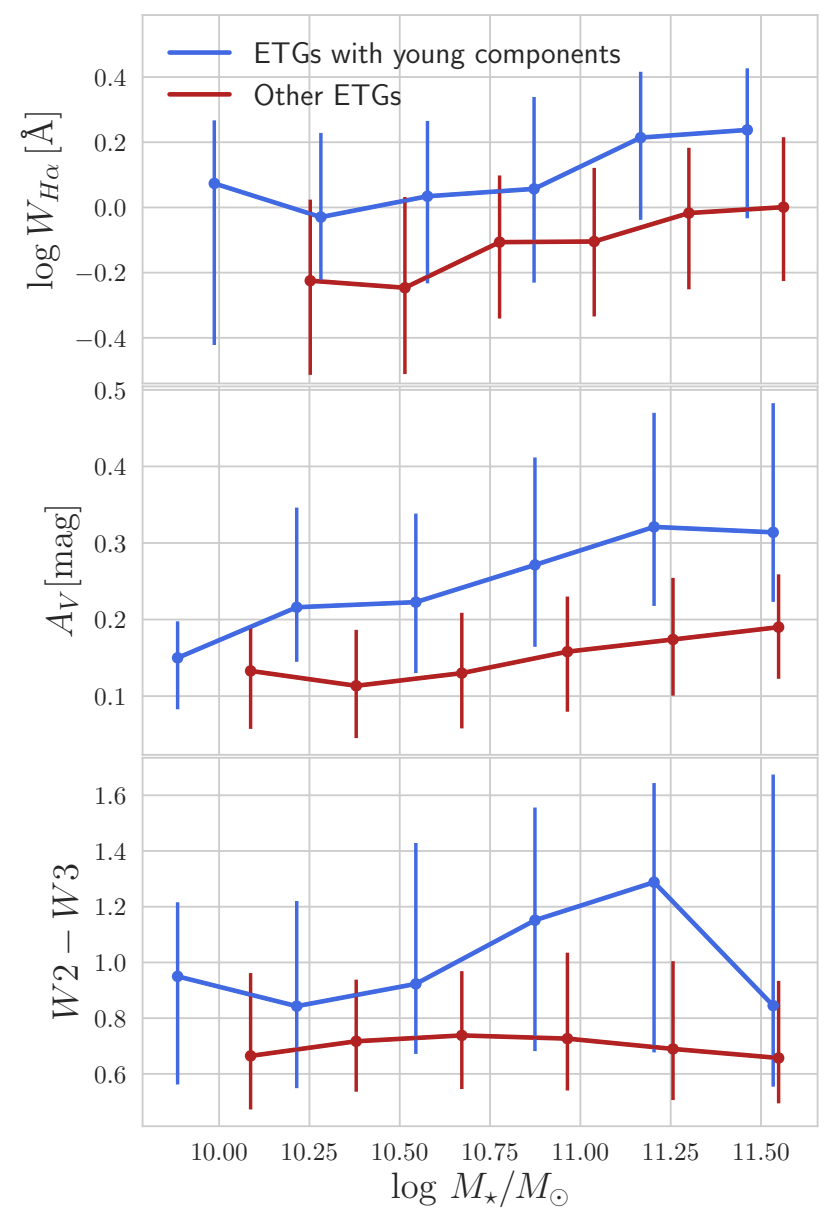

Figure 7. Median curves showing stellar mass $\left(\log M_{\star}\right)$ against $\mathrm{H} \alpha$ equivalent width $(\log W H \alpha$, top) and $V$-band dust attenuation $\left(A_{V}\right.$, middle) and $W 2-W 3$ color (bottom). Galaxies with and without young components are plotted in blue and red, respectively. Points are plotted in the centre of each $\log M_{\star}$ with error bars indicating the region between the percentiles of 25 and 75 per cent. In the top panel, we plot only galaxies with $S / N>3$ in $\mathrm{H} \alpha$, while in the bottom panel we exclude 3 galaxies for which there is no WISE data.

Herpich et al. (2018), who find that, at all stellar masses, ETGs with emission lines have larger $A_{V}$ and redder $W 2-W 3$ when compared to ETGs without emission lines.

We have shown that ETGs with young components have more ionised gas than other ETGs; however, the source of this ionisation is not clear. In Fig. 8 we plot the distribution of ETGs with young components in the WHAN (Cid Fernandes et al. 2011) and $\log [\mathrm{N} \mathrm{II}] \lambda 6584 / \mathrm{H} \alpha$ versus $\log \left[\mathrm{O}_{\mathrm{III}}\right] \lambda 5007 / \mathrm{H} \beta$ (named BPT after Baldwin, Phillips \& Terlevich 1981) diagrams, comparing them to other ETGs and to the general population of SDSS galaxies. In the BPT diagram, we plot the lines of Stasińska et al. (2006, solid line), Kauffmann et al. (2003, dashed line), Kewley et al. (2001, dot-dashed line) and the transposition of the Kewley et al. (2006) line proposed by Cid Fernandes et al. (2010, dotted line). We also indicate the galaxy classes usually defined using these diagrams. We only include in the diagrams objects that have signal to noise ratios $S / N>3$ in the re-
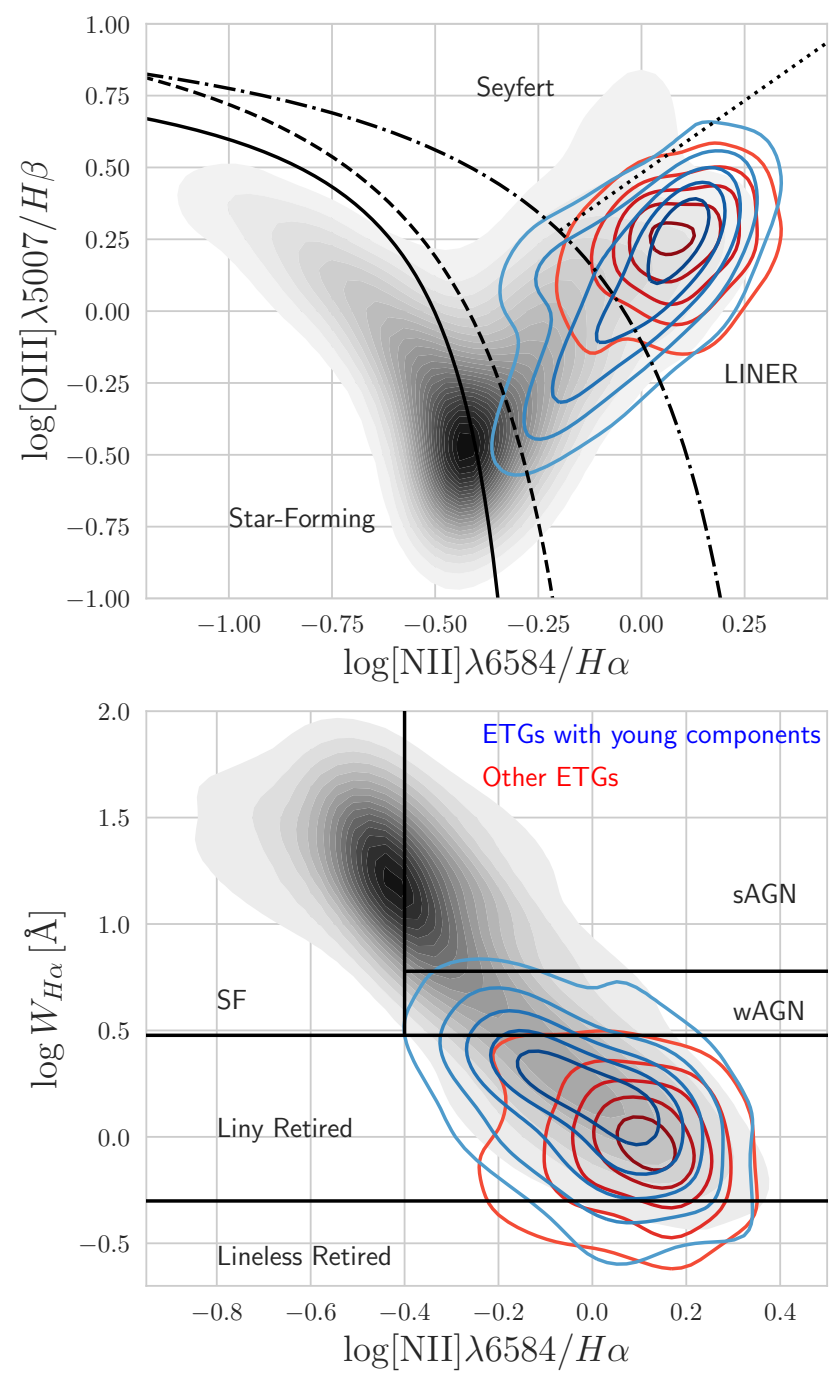

Figure 8. BPT (top) and WHAN (bottom) diagrams. ETGs with young components are shown as blue contours, other ETGs are shown in red. Only galaxies with $S / N>3$ in the lines used in each diagram are included. Filled contours in shades of gray show the distribution for the general sample DR7 galaxies that fit the same $S / N$ criteria. Contours represent a kernel density estimation with a Gaussian kernel.

quired lines. For the BPT, these are 33.3 per cent of ETGs with young components and 20.4 per cent of the rest of the sample. For the WHAN diagram, 65.3 per cent of ETGs with young components and 47.6 per cent of the rest of the sample satisfy the $S / N$ criterion.

The lines and classes indicated in Fig. 8 are useful to guide our analysis, but they should not be taken literally, as unresolved diagnostic diagrams tend to overlap multiple sources of ionisation (Sanchez 2019), and the galaxies studied in this paper are especially ambiguous in this regard. Objects above the line of Kewley et al. (2001) are often regarded as 'pure AGN'; however, this line represents how far objects can go when ionised only by $\mathrm{H}$ II regions. Thus, the interpretation is that above this line an object would need some ionisation mechanism other than star formation, although 
it does not mean that there is no star formation above this line. To add to the confusion, the Kewley et al. (2001) line was based on stellar atmosphere models that overestimated the hardness of the ionising field for $\mathrm{H}$ II regions. An updated version of the enveloping line for $\mathrm{H}$ II region photoionization models was provided by Stasińska et al. (2006); see also Dopita et al. (2013). Stasińska et al. (2006) estimate that $\sim 70$ per cent of the $\mathrm{H} \alpha$ flux at the Kewley et al. (2001) line may be due to young stars. Since galaxies studied in this paper are the oldest objects in the local universe, this estimate should be thought of as a conservative upper limit. Classes defined in the WHAN diagram are also misleading in the context of this work. Using integral field spectroscopy from the Calar Alto Legacy Integral Field spectroscopy Area survey (CALIFA, Sánchez et al. 2012), Lacerda et al. (2018) showed that combinations of $\mathrm{H}$ II regions and diffuse ionised gas may result in $W_{\mathrm{H} \alpha}$ and [ $\left.\mathrm{N} \mathrm{II}\right] \lambda 6584 / \mathrm{H} \alpha$ values typical of the AGN region in the WHAN diagram.

Most of ETGs studied in this paper lie in the LINER region of the BPT, but have low $\mathrm{H} \alpha$ equivalent width. Galaxies with these characteristics are classified as 'retired galaxies' or 'fake AGN' (Stasińska et al. 2008) and their dominant ionisation mechanism are HOLMES. For ETGs with young components, contours extend into the intermediate region of the BPT towards the star-forming wing, indicating an increasing contribution of young stars to the ionizing field. However, only one of these objects falls below the line of Stasińska et al. (2006), where ionisation can be accounted for solely by star formation. In the WHAN diagram, these contours extend towards larger values of $W_{\mathrm{H} \alpha}$, mostly in the region labelled as 'weak AGN' (wAGN). Thus we interpret that, at lower values of $[\mathrm{N} \mathrm{II}] \lambda 6584 / \mathrm{H} \alpha$, the ionisation in ETGs with young components likely comes from a mixture of young stars and HOLMES-powered diffuse ionised gas, as found by Lacerda et al. (2018). However, some contribution of AGN cannot be dismissed, and could be verified using integral field spectroscopy. As [N II] $\lambda 6584 / \mathrm{H} \alpha$ increases, the contribution of young stars decreases and HOLMES become the main ionising source. Regardless of the presence or absence of young components, 8 per cent of galaxies with BPT classification are above the Kewley et al. (2001) line and are also classified as AGN in the WHAN diagram. Thus, we do not see strong evidence for AGN feedback in our sample.

It is interesting to note that, although Herpich et al. (2018) find that ETGs with emission lines have slightly younger ages, they do not invoke young stellar populations as an ionising source. This apparent discrepancy can be easily understood as their work focused only on galaxies with $W_{\mathrm{H} \alpha}<3 \AA$, where HOLMES can solely account for all the ionizing radiation field. Since the rate of hydrogen ionizing photons in a young stellar population drops significantly after $10 \mathrm{Myr}$, and pinning down the age of the young stellar populations with the required precision is beyond what can be reliably achieved by our spectral synthesis method, we are unable to constrain the contribution young stars to the ionizing field from our synthesis results. Therefore, even in our sample we would not need to consider any ionization source other than HOLMES, were it not for the fact that many ETGs with young components have $W_{\mathrm{H} \alpha}$ values at the edge or above the threshold for retired galaxies and $[\mathrm{N}$ II] $]$ 6584/H $\alpha$ lower than expected for typical LINER-like emission. This makes the young + old stars scenario pro-

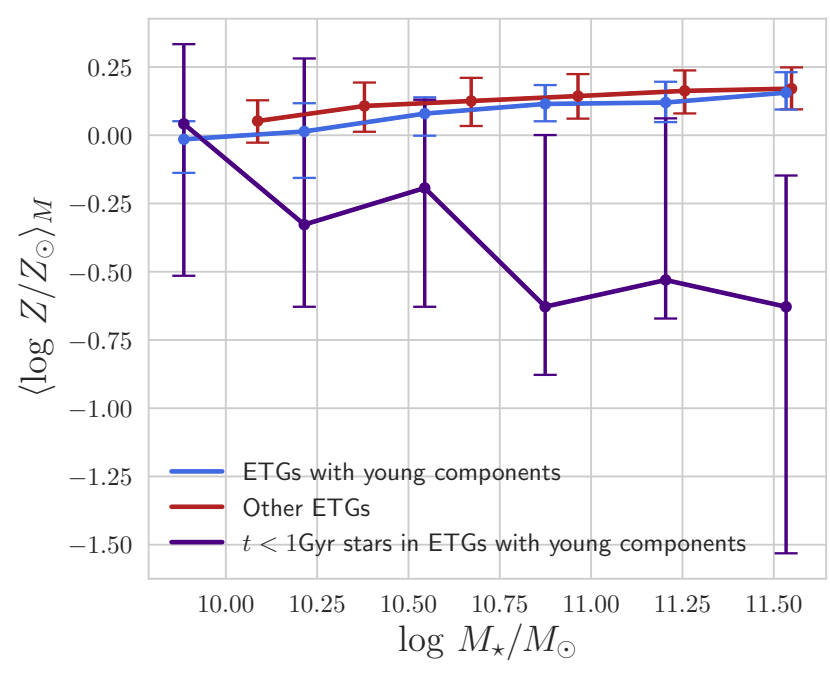

Figure 9. Median mass-weighted stellar metallicity against stellar mass. Red lines correspond to galaxies without young components. Blue and purple lines both correspond to ETGs with young components. While the blue line is the average metallicity of all stars in the galaxy, the purple line shows the metallicity of stellar populations younger than $1 \mathrm{Gyr}$. As in other figures, points are plotted in the centre of each stellar mass bin and error-bars represent the interquartile regions.

posed by Lacerda et al. (2018) the most likely one. Also, contributions from other ionizing sources such as AGN, binary stars or shocks cannot be completely ruled out.

\subsection{Where do young components come from?}

Although we have established that ETGs with young components have more dust and gas than other ETGs, and that their emission lines are consistent with recent star formation, this doesn't take us far in explaining how these young components have formed. Broadly speaking, these recent events of star-formation can be explained either by internal processes, where stars are formed from gas recycling consistent with a closed box model (e.g. Vazdekis et al. 1997) or by external processes such as minor mergers or cold gas accretion from the intergalactic medium. Of course, these are not mutually exclusive, and both effects are expected to play a role to some extent. In the first scenario, new stars form from gas that is already chemically enriched, so one expects the recently formed stars to be more metal-rich than the older stars in the galaxy. On the other hand, external processes would give origin to young components that are metal-poor. The absorption of a star-forming companion would add a population of young metal-poor stars to the galaxy and/or provide metal-poor gas from which new stars can form. Since gas in the intergalactic medium is expected to be metal-poor (see van de Voort \& Schaye 2012), stars formed from the accretion of this gas would also be more metal-poor than older stellar populations.

To test these hypotheses, in Fig. 9 we compare the massweighted mean stellar metallicity of the young components (purple lines) to the global (i.e. averaged over all stellar populations) values for ETGs with (blue) and without (red) young components. We emphasise that the blue and purple 
lines correspond to the same galaxies; while the blue line is averaged over all stellar populations, the purple one corresponds only to stars that are younger than 1 Gyr.

In terms of the global values (i.e. comparing the blue and red lines), there is very little difference in metallicity between ETGs with young components and the rest of our sample. However, it is clear that the young components are more metal-poor (i.e. comparing the purple to either the blue or red lines), and become more so with increasing stellar mass.

We interpret this as external processes becoming more relevant as stellar mass increases. At low stellar mass, galaxies have crossed the green valley more recently. In these systems, mass assembly is more gradual, and closed boxlike chemical enrichment might be still ongoing. Thus, some newly formed stars are more metal-rich than the older stellar populations. At larger stellar masses, most of the available gas in the ISM has already been turned into stars long ago, so external processes become the most likely (if not the only) path to the formation or accretion of new stars. Therefore, at large masses, it is likely that the young components are the result of rejuvenation (e.g. Pawlik et al. 2018; Chauke et al. 2019) events triggered by minor mergers or cold gas accretion in previously quiescent systems. A merger-driven scenario is consistent with results by Kaviraj et al. (2011), who find that ETGs at $z \sim 0.6$ showing signs of recent starformation are more likely to exhibit disturbed morphologies reminiscent of recent minor mergers. Our interpretation is also consistent with the results of Martin et al. (2017), who find that low-mass galaxies in the green valley are mostly quenching their star formation, while for high-mass galaxies there is a combination of quenching and bursting due to red sequence galaxies that become temporarily bluer after the triggering of star formation.

Additionally, at large stellar masses we should expect our sample to be dominated by slow rotators (see Emsellem et al. 2011), and it was found by Davis et al. (2011) that cold gas in these systems is misaligned with the stellar component, indicating the prevalence of external processes. In fast rotators, of typically lower mass, gas tends to be aligned with the stellar component, which is also compatible with the prevalence of internal processes for lower mass systems.

\subsection{Environment}

In this section, we investigate differences in environment between the sub-classes defined in section 4.1. To do so, we have matched our sample to the Yang et al. (2007) catalogue of galaxy groups. This catalogue is based on the method described by Yang et al. (2005) and contains estimates of dark matter halo masses and classifies galaxies as centrals or satellites.

Out of the 3453 ETGs in our sample, 3355 (97 per cent) are included in the Yang et al. (2007) catalogue. The vast majority of these (72.5 per cent) are central objects, while 27.5 per cent are satellites. There is a small variation in the proportion of centrals and satellites between the sub-classes defined in section 4.1. The fraction of centrals is 65.9 per cent for ETGs with young components, 76.9 per cent for UV upturn systems and 72.6 per cent for other ETGs, implying that galaxies with older stellar populations are more likely to be at the centre of dark matter halos.

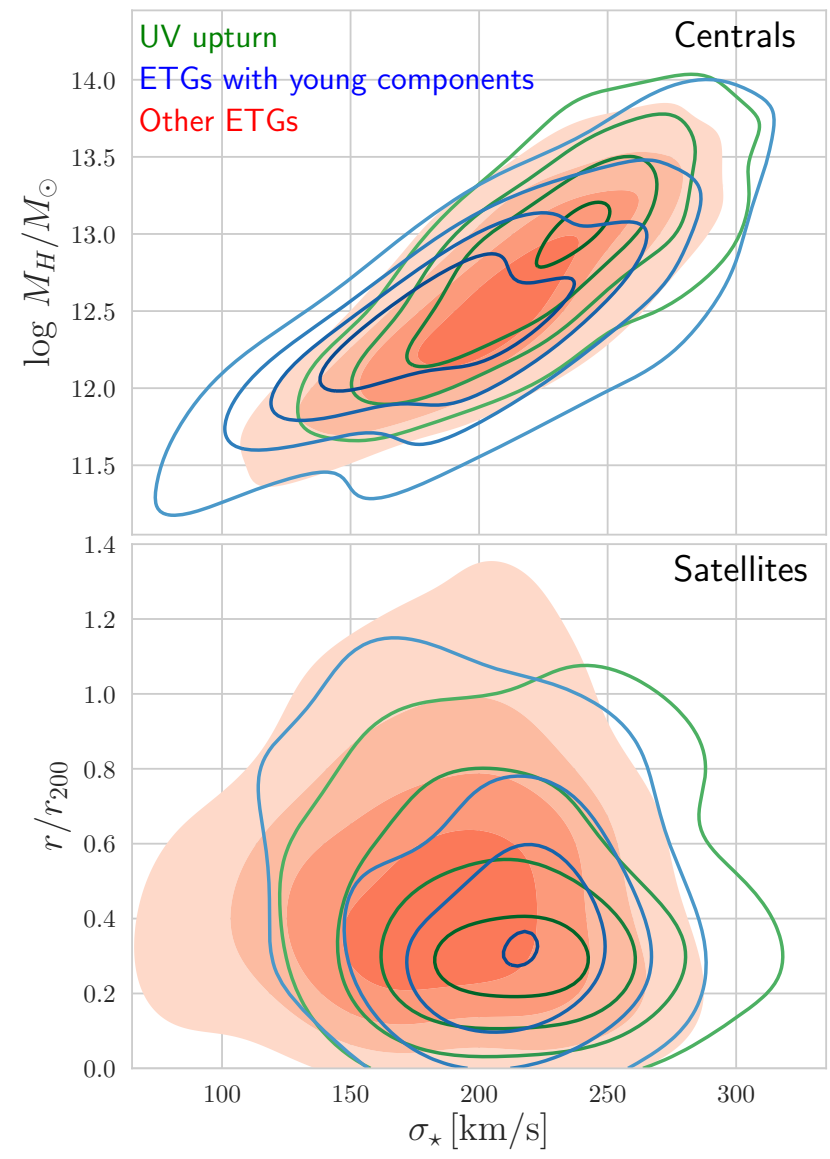

Figure 10. Top: Stellar velocity dispersion $\left(\sigma_{\star}\right)$ against halo mass $\left(\log M_{H}\right)$ for central galaxies. Bottom: $\sigma_{\star}$ against the projected distance to the luminosity-weighted centre of the dark matter halo (normalised by $r_{200}$ ) for satellite galaxies. Blue contours correspond to ETGs with young components, green contours correspond to UV upturn galaxies, and filled red contours in the background show the distribution for other ETGs. Contours trace a kernel density estimates calculated using a Gaussian kernel.

In Fig. 10 we plot parameters from the Yang et al. (2007) catalogue for galaxies in our sample. The top panel shows the distribution of halo mass $\left(\log M_{H}\right)$ against stellar velocity dispersion $\left(\sigma_{\star}\right)$ for central, while in the bottom panel of we plot $\sigma_{\star}$ against the luminosity-weighted distance to the centre of the dark matter halo, normalised by $r_{200}$. Contours show kernel density estimates for ETGs with young components (blue), UV upturn systems (green) and other ETGs (red). For central galaxies (top panel in Fig. 10), we see clear differences in $\log M_{H}$ between the subclasses: ETGs with young components are more common in low-mass halos, UV upturn systems are in more massive halos and other ETGs represent an intermediate case. For satellites (bottom panel in Fig. 10), the differences in environment between the sub-classes are unclear.

Comparing the two panels of Fig. 10, one notices that ETGs with young components are mostly in the low- $\sigma_{\star}$ end of the distribution for centrals and in the high- $\sigma_{\star}$ end for satellites. One also notices that, at lower masses, central ETGs with young components tend to have slightly larger halo masses than other central galaxies in our sample. This 
is consistent with our interpretation of Fig. 9. In low-mass halos, galaxies are more isolated, making external processes less likely. Also, star formation in these systems will be facilitated in higher mass halos, where it is easier to retain gas in the circumgalactic medium. Further away from the halo's centre, galaxies have to rely on their own dynamical masses in order to fuel star formation. Thus, star formation in satellites is restricted to the most massive galaxies, as seen in the middle panel of Fig. 10.

UV upturn systems require larger fractions of older stellar populations to reproduce their UV emission. Thus, these objects are expected to have assembled their stellar mass over short timescales at an early epoch. Here we show that these systems belong to denser environments than galaxies that assembled their stellar mass more gradually. These results are compatible with the top-down mass assembly scenario, where high-mass galaxies assembled their stellar mass earlier (Cimatti et al. 2006).

\section{CONCLUSIONS}

In this work, we have used state-of-the-art stellar population models to simultaneously fit SDSS spectra and GALEX photometry of a sample of 3453 early-type galaxies galaxies in the red sequence $(N U V-r>5)$ at $z<0.1$. We use the STARLIGHT spectral synthesis code to model the integrated light of the galaxies as a non-parametric combination of stellar population spectra. Our main results are the following:

- The distributions of light- and mass-weighted mean stellar ages broaden significantly with the addition of UV data. This broadening is observed to a smaller extent in metallicity and dust attenuation. The trend is consistent with the fact that the distribution of colours in the red sequence is broader in the UV than in the optical;

- We are able to reproduce the FUV magnitudes within error bars for 80 per cent of UV upturn galaxies selected with the Yi et al. (2011) criteria $(N U V-r>5.4, F U V-N U V<0.9$ and $F U V-r<6.6)$. This suggests that additional ingredients like binaries or EHB stars such as the ones observed by Brown et al. (2000a) in M32 should have a limited contribution in the overall population of UV upturn systems;

- As in previous works (e.g. Dantas et al. 2020; Le Cras et al. 2016b), we find that UV upturn galaxies are more massive and have redder $N U V-r$ colours than other ETGs;

- In qualitative agreement with previous work (e.g. Kaviraj et al. 2007), we find that a significant fraction (43.3 per cent) of galaxies with $5<N U V-r<5.5$ show some contribution of stellar populations younger than $1 \mathrm{Gyr}$. For $N U V-r>5.5$ the fraction drops to 9.5 per cent;

- Regardless of stellar mass, ETGs with young components have more dust and ionised gas than other ETGs, which is in agreement with Herpich et al. (2018). We interpret the source of ionising photons in these systems as a mixture of young stars and HOLMES, while HOLMES account for most of the ionisation in other ETGs.

- The young components that we detect are of low metallicity, and increasingly so for larger stellar masses. This indicates that external processes such as mergers and accretion of cold gas from the intergalactic medium play a larger role in triggering star formation in high-mass galaxies, while in low-mass systems (that are still making their way into the red sequence) new stars can be formed from gas recycling as if in a closed box model (e.g. Vazdekis et al. 1997);

- When examining galaxies at the centres of dark matter halos, we find that ETGs with young components are more common in low-mass halos, UV upturn systems reside in high-mass halos and other ETGs fall in an intermediate case. For satellites, the differences in environment between these sub-classes are unclear.

The combination of detailed $\lambda$-by- $\lambda$ information from optical spectra and the unique constraints provided by UV data have allowed us to improve upon previous works modelling the stellar populations of ETGs. However, modelling of UV-optical emission in these systems is challenging, and the work presented here is subject to some limitations. The stellar population models used in this work are still incomplete and could benefit from adding additional ingredients such as binaries and EHB stars. Other limitation comes from our treatment of star-dust geometry. We work under the assumption that all stars are attenuated by the same dust optical depth, when in reality young stars are more strongly affected by dust. Better modelling could provide further clues in the recent and early history of ETGs, and thus contribute to our understanding these systems and of galaxy evolution in general.

\section{ACKNOWLEDGEMENTS}

We thank the anonymous referee, as well as the scientific editor, for their clear and relevant suggestions. AW thanks Maria Luiza Linhares Dantas and Roderik Overzier for important discussions about this paper. AW acknowledges financial support from Fundação de Amparo à Pesquisa do Estado de São Paulo (FAPESP) process number 2019/01768-6. $\mathrm{RCF}$ acknowledges financial suport from Conselho Nacional de Desenvolvimento Científico e Tecnológico (CNPq) (grant 302270/2018-3) NVA acknowledges support of the Royal Society and the Newton Fund via the award of a Royal SocietyNewton Advanced Fellowship (grant NAF $\backslash R 1 \backslash 180403$ ), and of Fundação de Amparo à Pesquisa e Inovação de Santa Catarina (FAPESC) and Conselho Nacional de Desenvolvimento Científico e Tecnológico (CNPq). PC acknowledges financial support from Fundação de Amparo à Pesquisa do Estado de São Paulo (FAPESP) process number 2018/053928 and Conselho Nacional de Desenvolvimento Científico e Tecnológico (CNPq) process number 310041/2018-0. GB acknowledges financial support from the National $\mathrm{Au}-$ tonomous University of México (UNAM) through grant DGAPA/PAPIIT IG100319 and from CONACyT through grant CB2015-252364. RRdC acknowledges financial support from FAPESP through the grant 2014/111564. LSJ acknowledges support from Brazilian agencies CNPq (grant 304819/2017-4) and FAPESP (grant 2012/00800-4). Funding for the SDSS and SDSS-II has been provided by the Alfred P. Sloan Foundation, the Participating Institutions, the National Science Foundation, the U.S. Department of Energy, the National Aeronautics and Space Administration, the Japanese Monbukagakusho, the Max Planck Society, and the Higher Education Funding Council for England. The SDSS Web Site is http://www.sdss.org/. The SDSS is managed by the Astrophysical Research Consortium for the Participating Institutions. The Participating Institutions 
are the American Museum of Natural History, Astrophysical Institute Potsdam, University of Basel, University of Cambridge, Case Western Reserve University, University of Chicago, Drexel University, Fermilab, the Institute for Advanced Study, the Japan Participation Group, Johns Hopkins University, the Joint Institute for Nuclear Astrophysics, the Kavli Institute for Particle Astrophysics and Cosmology, the Korean Scientist Group, the Chinese Academy of Sciences (LAMOST), Los Alamos National Laboratory, the Max-Planck-Institute for Astronomy (MPIA), the MaxPlanck-Institute for Astrophysics (MPA), New Mexico State University, Ohio State University, University of Pittsburgh, University of Portsmouth, Princeton University, the United States Naval Observatory, and the University of Washington. This project made use of GALEX data and the Barbara A. Mikulski Archive for Space Telescopes. This research made use of Astropy, ${ }^{3}$ a community-developed core Python package for Astronomy (Astropy Collaboration et al. 2013; Price-Whelan et al. 2018).

\section{DATA AVAILABILITY}

The data underlying this article will be shared on reasonable request to the corresponding author.

\section{REFERENCES}

Abazajian K. N., et al., 2009, ApJS, 182, 543

Agius N. K., et al., 2013, MNRAS, 431, 1929

Astropy Collaboration et al., 2013, A\&A, 558, A33

Baldwin J. A., Phillips M. M., Terlevich R., 1981, PASP, 93, 5

Bica E., Alloin D., 1987, A\&AS, 70, 281

Blanton M. R., Roweis S., 2007, AJ, 133, 734

Bressan A., Marigo P., Girardi L., Salasnich B., Dal Cero C., Rubele S., Nanni A., 2012, MNRAS, 427, 127

Brown T. M., Bowers C. W., Kimble R. A., Ferguson H. C., 2000a, ApJ, 529, L89

Brown T. M., Bowers C. W., Kimble R. A., Sweigart A. V., Ferguson H. C., 2000b, ApJ, 532, 308

Bruzual G., Charlot S., 2003, MNRAS, 344, 1000

Burstein D., Bertola F., Buson L. M., Faber S. M., Lauer T. R., 1988, ApJ, 328, 440

Caldwell N., Rose J. A., Concannon K. D., 2003, AJ, 125, 2891

Calzetti D., Kinney A. L., Storchi-Bergmann T., 1994, ApJ, 429, 582

Calzetti D., Armus L., Bohlin R. C., Kinney A. L., Koornneef J., Storchi-Bergmann T., 2000, ApJ, 533, 682

Cappellari M., et al., 2011, MNRAS, 416, 1680

Cappellari M., et al., 2013, MNRAS, 432, 1862

Cardelli J. A., Clayton G. C., Mathis J. S., 1989, ApJ, 345, 245

Carleton T., Guo Y., Nayyeri H., Cooper M., Rudnick G., Whitaker K., 2020, MNRAS, 491, 2822

Castignani G., Pandey-Pommier M., Hamer S. L., Combes F., Salomé P., Freundlich J., Jablonka P., 2020, arXiv e-prints, p. arXiv:2004.01786

Chabrier G., 2003, PASP, 115, 763

Charlot S., Fall S. M., 2000, ApJ, 539, 718

Chauke P., et al., 2019, The Astrophysical Journal, 877, 48

Chen Y., Bressan A., Girardi L., Marigo P., Kong X., Lanza A., 2015, MNRAS, 452, 1068

Cid Fernandes R., 2007, arXiv e-prints, pp astro-ph/0701902

3 http://www.astropy.org
Cid Fernandes R., González Delgado R. M., 2010, MNRAS, 403, 780

Cid Fernandes R., Mateus A., Sodré L., Stasińska G., Gomes J. M., 2005, MNRAS, 358, 363

Cid Fernandes R., Stasińska G., Schlickmann M. S., Mateus A., Vale Asari N., Schoenell W., Sodré L., 2010, MNRAS, 403, 1036

Cid Fernandes R., Stasińska G., Mateus A., Vale Asari N., 2011, MNRAS, 413, 1687

Cid Fernandes R., et al., 2013, A\&A, 557, A86

Cid Fernandes R., et al., 2014, A\&A, 561, A130

Cimatti A., Daddi E., Renzini A., 2006, A\&A, 453, L29

Code A. D., Welch G. A., 1979, ApJ, 228, 95

Conroy C., 2013, ARA\&A, 51, 393

Dantas M. L. L., Coelho P. R. T., de Souza R. S., Gonçalves T. S., 2020, MNRAS, 492, 2996

Davis T. A., et al., 2011, MNRAS, 417, 882

Dawson K. S., et al., 2013, AJ, 145, 10

Deharveng J. M., Boselli A., Donas J., 2002, A\&A, 393, 843

Dopita M. A., Sutherland R. S., Nicholls D. C., Kewley L. J., Vogt F. P. A., 2013, ApJS, 208, 10

Dorman B., Rood R. T., O'Connell R. W., 1993, ApJ, 419, 596

Eldridge J. J., Stanway E. R., 2012, MNRAS, 419, 479

Emsellem E., et al., 2007, MNRAS, 379, 401

Emsellem E., et al., 2011, MNRAS, 414, 888

Flores-Fajardo N., Morisset C., Stasińska G., Binette L., 2011, MNRAS, 415, 2182

Gonçalves T. S., Martin D. C., Menéndez-Delmestre K., Wyder T. K., Koekemoer A., 2012, ApJ, 759, 67

Greggio L., Renzini A., 1990, ApJ, 364, 35

Hamann W. R., Gräfener G., 2004, A\&A, 427, 697

Han Z., Podsiadlowski P., Lynas-Gray A. E., 2007, Monthly Notices of the Royal Astronomical Society, 380, 1098

Hernández-Pérez F., Bruzual G., 2014, MNRAS, 444, 2571

Herpich F., Stasińska G., Mateus A., Vale Asari N., Cid Fernandes R., 2018, MNRAS, 481, 1774

Janowiecki S., Catinella B., Cortese L., Saintonge A., Wang J., 2020, arXiv e-prints, p. arXiv:2001.06614

Jeong H., et al., 2009, MNRAS, 398, 2028

Kauffmann G., et al., 2003, MNRAS, 346, 1055

Kauffmann G., et al., 2007, ApJS, 173, 357

Kaviraj S., Devriendt J. E. G., Ferreras I., Yi S. K., 2005, MNRAS, 360, 60

Kaviraj S., et al., 2007, ApJS, 173, 619

Kaviraj S., Tan K.-M., Ellis R. S., Silk J., 2011, MNRAS, 411, 2148

Kewley L. J., Dopita M. A., Sutherland R. S., Heisler C. A., Trevena J., 2001, ApJ, 556, 121

Kewley L. J., Groves B., Kauffmann G., Heckman T., 2006, MNRAS, 372, 961

Knapp G. R., 1999, in Carral P., Cepa J., eds, Astronomical Society of the Pacific Conference Series Vol. 163, Star Formation in Early Type Galaxies. p. 119 (arXiv:astro-ph/9808266)

La Barbera F., Lopes P. A. A., de Carvalho R. R., de La Rosa I. G., Berlind A. A., 2010, MNRAS, 408, 1361

La Barbera F., Pasquali A., Ferreras I., Gallazzi A., de Carvalho R. R., de la Rosa I. G., 2014, MNRAS, 445, 1977

Lacerda E. A. D., et al., 2018, MNRAS, 474, 3727

Lanz T., Hubeny I., 2003a, ApJS, 146, 417

Lanz T., Hubeny I., 2003b, ApJS, 147, 225

Lanz T., Hubeny I., 2007, ApJS, 169, 83

Larson R. B., 1974, MNRAS, 166, 585

Le Cras C., Maraston C., Thomas D., 2016a, IAU Focus Meeting, 29B, 190

Le Cras C., Maraston C., Thomas D., York D. G., 2016b, MNRAS, 461, 766

Leitherer C., Li I.-H., Calzetti D., Heckman T. M., 2002, The Astrophysical Journal Supplement Series, 140, 303 
Leitherer C., Ortiz Otálvaro P. A., Bresolin F., Kudritzki R.-P., Lo Faro B., Pauldrach A. W. A., Pettini M., Rix S. A., 2010, ApJS, 189, 309

Lintott C. J., et al., 2008, MNRAS, 389, 1179

López Fernández R., et al., 2016, MNRAS, 458, 184

Martin D. C., et al., 2005, ApJ, 619, L1

Martin D. C., Gonçalves T. S., Darvish B., Seibert M., Schiminovich D., 2017, ApJ, 842, 20

Martins L. P., González Delgado R. M., Leitherer C., Cerviño M., Hauschildt P., 2005, MNRAS, 358, 49

Mateus A., Sodré L., Cid Fernandes R., Stasińska G., Schoenell W., Gomes J. M., 2006, MNRAS, 370, 721

Miller Bertolami M. M., 2019, in Kerschbaum F., Groenewegen M., Olofsson H., eds, IAU Symposium Vol. 343, IAU Symposium. pp 36-46, doi:10.1017/S1743921318007330

O'Connell R. W., 1999, ARA\&A, 37, 603

Ocvirk P., 2010, ApJ, 709, 88

Pawlik M. M., et al., 2018, MNRAS, 477, 1708

Penoyre Z., Moster B. P., Sijacki D., Genel S., 2017, MNRAS, 468,3883

Petty S. M., et al., 2013, AJ, 146, 77

Price-Whelan A. M., et al., 2018, AJ, 156, 123

Rauch T., 2003, A\&A, 403, 709

Rowlands K., et al., 2018, MNRAS, 473, 1168

Salvador-Rusiñol N., Vazdekis A., La Barbera F., Beasley M. A., Ferreras I., Negri A., Dalla Vecchia C., 2019, Nature Astronomy, p. 1

Sanchez S. F., 2019, arXiv e-prints, p. arXiv:1911.06925

Sánchez-Blázquez P., et al., 2006, MNRAS, 371, 703

Sánchez S. F., et al., 2012, A\&A, 538, A8

Schawinski K., et al., 2007, ApJS, 173, 512

Schlafly E. F., Finkbeiner D. P., 2011, ApJ, 737, 103

Schlegel D. J., Finkbeiner D. P., Davis M., 1998, ApJ, 500, 525

Simonian G. V., Martini P., 2016, Monthly Notices of the Royal Astronomical Society, 464, 3920

Smethurst R. J., et al., 2018, MNRAS, 473, 2679

Smith R. J., Lucey J. R., Carter D., 2012, Monthly Notices of the Royal Astronomical Society, 421, 2982

Stasińska G., Cid Fernandes R., Mateus A., Sodré L., Asari N. V., 2006, MNRAS, 371, 972

Stasińska G., et al., 2008, MNRAS, 391, L29

Valdes F., Gupta R., Rose J. A., Singh H. P., Bell D. J., 2004, ApJS, 152, 251

Vassiliadis E., Wood P. R., 1993, ApJ, 413, 641

Vassiliadis E., Wood P. R., 1994, ApJS, 92, 125

Vazdekis A., Peletier R. F., Beckman J. E., Casuso E., 1997, ApJS, 111, 203

Vazdekis A., Koleva M., Ricciardelli E., Röck B., Falcón-Barroso J., 2016, MNRAS, 463, 3409

Walcher J., Groves B., Budavári T., Dale D., 2011, Ap\&SS, 331, 1

Welch G. A., Sage L. J., Young L. M., 2010, The Astrophysical Journal, 725, 100

Werle A., Cid Fernandes R., Vale Asari N., Bruzual G., Charlot S., Gonzalez Delgado R., Herpich F. R., 2019, MNRAS, 483, 2382

Wright E. L., et al., 2010, AJ, 140, 1868

Yang X., Mo H. J., van den Bosch F. C., Jing Y. P., 2005, MNRAS, 356, 1293

Yang X., Mo H. J., van den Bosch F. C., Pasquali A., Li C., Barden M., 2007, ApJ, 671, 153

Yi S. K., 2008, in Heber U., Jeffery C. S., Napiwotzki R., eds, Astronomical Society of the Pacific Conference Series Vol. 392, Hot Subdwarf Stars and Related Objects. p. 3 (arXiv:0808.0254)

Yi S., Demarque P., Oemler Augustus J., 1997, ApJ, 486, 201

Yi S. K., et al., 2005, ApJ, 619, L111
Yi S. K., Lee J., Sheen Y.-K., Jeong H., Suh H., Oh K., 2011, ApJS, 195, 22

York D. G., et al., 2000, AJ, 120, 1579

Young L. M., et al., 2014, Monthly Notices of the Royal Astronomical Society, 444, 3408

van de Voort F., Schaye J., 2012, Monthly Notices of the Royal Astronomical Society, 423, 2991

This paper has been typeset from a $\mathrm{T}_{\mathrm{E} X} / \mathrm{LAT}_{\mathrm{E}} \mathrm{X}$ file prepared by the author. 